\title{
Channel Waveguide Lasers Produced by Femtosecond and Picosecond Direct Laser Writing in Ti:Sapphire Crystals
}

\author{
C. Grivas ${ }^{1, *}$, C. Corbari ${ }^{2}$, G. Brambilla ${ }^{2}$, P. G. Kazansky ${ }^{2}$, and P. G. Lagoudakis ${ }^{1}$ \\ ${ }^{1}$ School of Physics and Astronomy, University of Southampton, Southampton SO17 1BJ, United Kingdom \\ ${ }^{2}$ Optoelectronics Research Centre, University of Southampton, Southampton SO17 1BJ, United Kingdom \\ chr.grivas@gmail.com
}

\begin{abstract}
Femtosecond-laser-written Ti:sapphire channel waveguides lase at $\sim 798.25 \mathrm{~nm}$ above a threshold pump power of $84 \mathrm{~mW}$ with output power and slope efficiency of $143 \mathrm{~mW}$ and $23.5 \%$, respectively. Lasing was also observed in picosecond-laser-written channels.

OCIS codes: (140.3590) Lasers, Titanium; (230.7380) Waveguides, channeled
\end{abstract}

\section{Introduction}

Femtosecond (fs) lasers have emerged as a powerful tool for rapid prototyping of channel waveguide lasers and amplifiers in the bulk of different types of transparent glasses, crystals and more recently ceramic materials [1,2]. This approach allows for development of integrated photonic devices by side-stepping conventional photolithographic processes. Since for the vast majority of crystals exposure to the scanning laser beam results in negative refractive index changes, waveguide writing relies on engineering either depressed-cladding structures or two parallel tracks to confine the mode in the spacing in-between, by stress-induced increases in the refractive index.

Ti:sapphire $\left(\alpha-\mathrm{Al}_{2} \mathrm{O}_{3}: \mathrm{Ti}^{3+}\right)$ with its broad emission bandwidth $(650-1100 \mathrm{~nm})$ is a benchmark solid-state gain medium for ultrashort laser pulse generation and broadly tunable lasers. Ti:sapphire channel waveguides offer scope for both development of small footprint sources and reduction of the high pump powers required for laser operation in the bulk crystal due to the low peak emission cross section and short fluorescence lifetime of the $\mathrm{Ti}^{3+}$ ions [3].

Here, we report on the continuous wave (cw) laser operation of Ti:sapphire channel waveguides fabricated by fsand picosecond (ps) laser writing. To the best of our knowledge there has not been any report to date on either laserwritten Ti:sapphire waveguide lasers or any channel waveguide laser fabricated by ps-laser writing.

\section{Experimental details and channel waveguide fabrication}

Waveguide formation with femtosecond pulses was realized at a wavelength of $1030 \mathrm{~nm}$ using mode-locked regenerative amplified $\mathrm{Yb}: \mathrm{KGW}$ lasers operating at two different pulse duration/repetition regimes, namely, $180 \mathrm{fs} /$ $1 \mathrm{KHz}$ and $320 \mathrm{fs} / 200 \mathrm{kHz}$ respectively. In the picosecond regime, a Nd: $\mathrm{YVO}_{4}$ laser emitting a 200- $\mathrm{kHz}$ train of 8-ps-short pulses at $1064 \mathrm{~nm}$ was employed. The Ti:sapphire host crystal, uniformly doped with $0.12 \mathrm{wt} \% \mathrm{Ti}_{2} \mathrm{O}_{3}$, was mounted on a 3-D nano-translation stage, which allowed precise control of its position relative to the laser beam. Pairs of parallel tracks with separations that ranged from 15 to $24 \mu \mathrm{m}$ were fabricated along the crystal by focusing the laser pulses $\sim 150 \mu \mathrm{m}$ below the surface using a microscope objective lens with a numerical aperture (NA) of 0.65. Visual confirmation of focusing was provided by means of a CCD camera by imaging through the microscope objective. Writing was completed by scanning the sample transversally to the incident pulses in the same direction for the two tracks of the pair structure [4]. The processing scanning speeds $v_{s c}$ and laser energies $E_{p}$ depended on the laser used for writing and were as follows: (i) $v_{s c}=15 \mu \mathrm{m} \cdot \mathrm{s}^{-1}, E_{p}=1.5 \mu \mathrm{J},(180 \mathrm{fs}, 1 \mathrm{kHz}, 1030$ nm), (ii) $v_{s c}=2 \mathrm{~mm} \cdot \mathrm{s}^{-1}, E_{p}=0.065 \mathrm{~mJ}(320 \mathrm{fs}, 200 \mathrm{kHz}, 1030 \mathrm{~nm})$, and (iii) $v_{s c}=0.5 \mathrm{~mm} \cdot \mathrm{s}^{-1}, E_{p}=0.3 \mathrm{~mJ}(8 \mathrm{ps}, 200$ $\mathrm{kHz}, 1064 \mathrm{~nm}$ ). The laser polarization was set parallel to the writing direction of the sample.

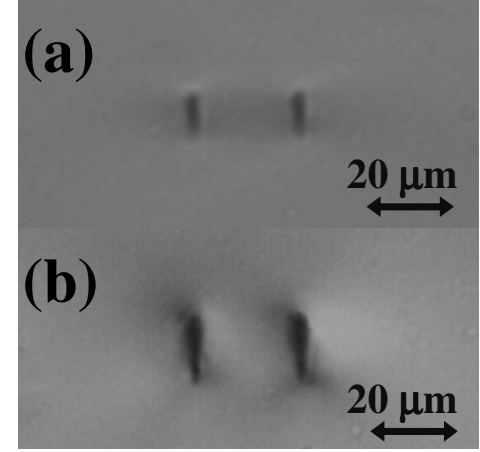

Fig. 1. Microscope images of two pairs of waveguide-defining tracks produced by (a) fs-pulses and (b) ps-pulses ( $8 \mathrm{ps}, 200 \mathrm{kHz}, 1064 \mathrm{~nm})$

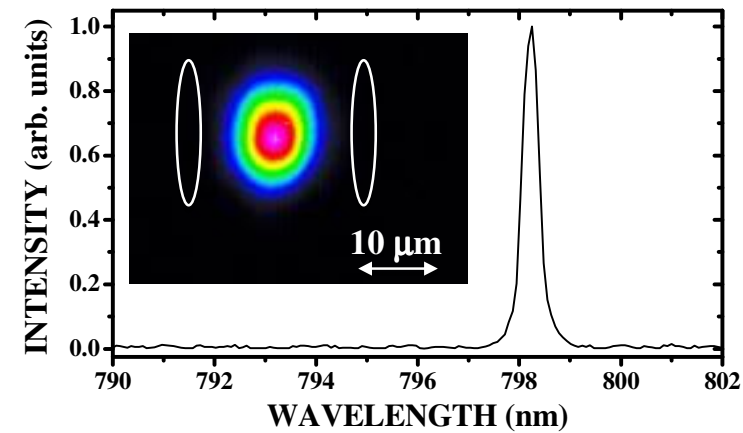

Fig. 2. Laser spectrum from a waveguide defined by two fs-laserwritten tracks with a spacing of $24 \mu \mathrm{m}$. In the inset, the mode intensity profile with the location of the tracks indicated by two ovals. 


\section{Laser operation}

Fig 1a and $1 \mathrm{~b}$ show two waveguides written by a fs-laser and ps-laser, respectively, both operating at $200 \mathrm{kHz}$, where it can be seen that the tracks produced by ps-pulses had a depth and width that were larger by a factor of $\sim 2.5$ Waveguide characterization was carried out using a diode-pumped solid-state (DPSS) pump laser emitting at 532 $\mathrm{nm}$. The fluorescence spectra from the waveguides were similar to that of the unprocessed crystal, indicating the absence of any fluorescence quenching of the $\mathrm{Ti}^{3+}$ ions by irradiation-induced stress.

The laser cavity was formed by attaching two high-reflective $(\mathrm{HR})$ mirrors $(\mathrm{R}=99.5 \%)$ at the signal wavelength at the end-faces of the 4-mm-long waveguides. Figure 2 displays a laser spectrum from a waveguide defined by two tracks machined by fs-laser-pulses $(180 \mathrm{fs}, 1 \mathrm{kHz}, 1030 \mathrm{~nm})$ at a spacing of $24-\mu \mathrm{m}$. It was recorded by an optical spectrum analyzer (OSA) and exhibits an emission peak at a wavelength of $798.25 \mathrm{~nm}$. Laser oscillation for this waveguide was obtained above a threshold of $\mathrm{P}_{\mathrm{th}}=84 \mathrm{~mW}$ of absorbed pump power, which represents a reduction by a factor of $>3$ in comparison to their counterparts produced by other methods [4]. The laser output was $\pi$ polarized regardless of the polarization state of the pump beam due to the larger emission cross section of the waveguide for the $\pi$ compared to the $\sigma$ polarization. Also shown in Fig 2, is an intensity profile of the laser mode, as captured by a CCD camera, indicating that the guides can produce a strong optical confinement. The laser output characteristics were studied using a set of three outcoupling mirrors with a transmission of $2 \%, 10 \%$, and $35 \%$ at the laser wavelength. The maximum output power, $143 \mathrm{~mW}$ for about $1 \mathrm{~W}$ of absorbed pump power and highest slope efficiency, $\eta=23.5 \%$ were obtained with $35 \%$ outcoupling (Fig. 3). Waveguides with the same spacing characteristics produced by fs-laser pulse trains at higher repletion rates $(200 \mathrm{kHz})$ consistently exhibited $\sim 20 \%$ lower output powers for the same outcoupling level, most likely as a result of weaker mode confinement.

Lasing in a waveguide based on a pair of $24-\mu \mathrm{m}$ spaced, ps-laser-written tracks was obtained above a pump threshold of $189 \mathrm{~mW}$. The power characteristics of this source for outcoupling levels of $2 \%$ and $10 \%$, is shown in Fig 4. The lower maximum output powers $(45 \mathrm{~mW})$ and slopes efficiencies $(7.1 \%)$ obtained for this writing regime are due to the stronger interaction of the modal field with the tracks. Using a model that describes the effects of transverse mode profiles on $\eta$ in longitudinally pumped lasers [5], an upper loss of 0.6 and $2 \mathrm{dBcm}^{-1}$ was derived from the $\eta$ values obtained for the fs- and ps-laser written waveguides, respectively. As these values also account for losses due to imperfect cavity mirrors attachment and end face polishing propagation loss should be lower.

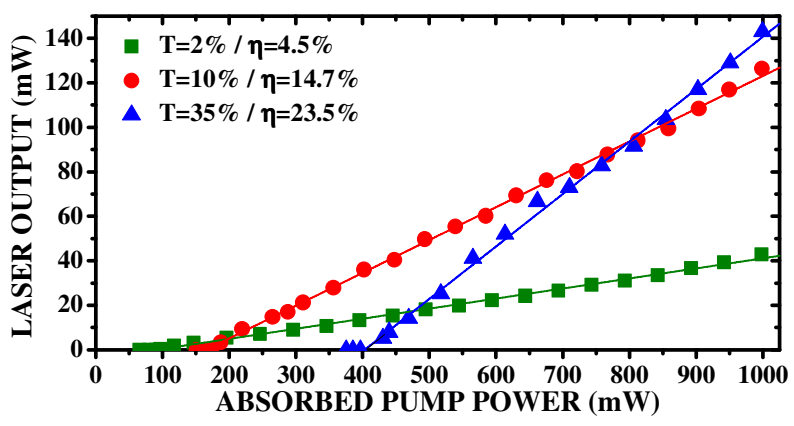

Fig. 3. Power characteristics for a Ti:sapphire channels waveguide laser produced by fs-laser writing of two tracks with a $24-\mu \mathrm{m}$-spacing.

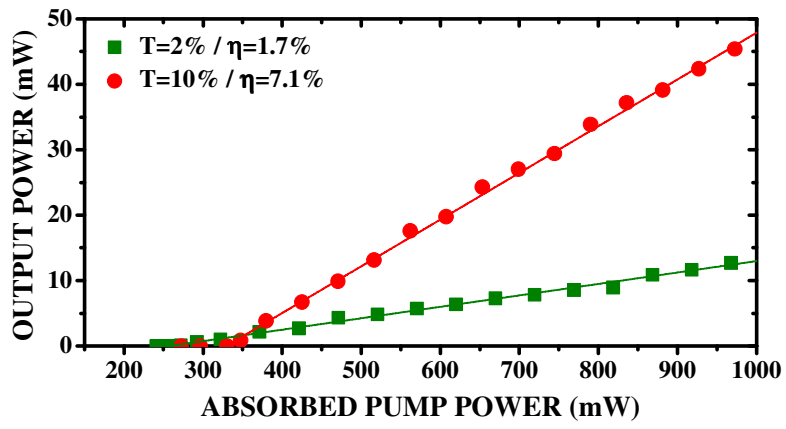

Fig. 4. Output power dependence on pump power for waveguide laser defined by a pair of 24- $\mu \mathrm{m}$-spaced tracks inscribed by ps-laser.

\section{Conclusions}

$\mathrm{CW}$ lasing above a pump threshold of $84 \mathrm{~mW}$ was demonstrated in channel waveguides written in a Ti:sapphire crystal with fs-laser pulses. To the best of our knowledge, they produced the highest output power $(143 \mathrm{~mW})$ and slope efficiency (23.5\%) of any Ti:sapphire channel waveguide laser to date, offering prospects for development of mode-locked sources. In the same material, we also demonstrated the first waveguide laser inscribed by ps-pulses.

\section{References}

[1] M. Ams, G. D. Marshall, P. Dekker, J. A. Piper and M. J. Withford "Ultrafast laser written active devices," Laser Photon. Rev. 3, 535-544 (2009).

[2] C. Grivas "Optically pumped planar waveguide lasers, Part I: Fundamentals and fabrication techniques," Prog. Quantum Electron. 35, 159-239 (2011).

[3] W. Yang, P. G. Kazansky, Y. P. Svirko “Non-reciprocal ultrafast laser writing” Nature Photon. 2, 99-104 (2008)

[4] M. Pollnau, C. Grivas, L. Laversenne, J. S. Wilkinson, R. W. Eason, and D. P. Shepherd, "Ti:sapphire waveguide lasers," Laser Phys. Lett. 4, 560-571, (2007).

[5] W. A. Clarkson and D. C. Hanna, "Effects of transverse-mode Profile on slope efficiency and relaxation oscillations in a longitudinally-pumped laser," J. Mod. Opt. 36, 483-498 (1989) 